\title{
Correction to: Germinal Immunogenetics predict treatment outcome for PD-1/PD-L1 checkpoint inhibitors
}

\author{
Sadal Refae ${ }^{1} \cdot$ Jocelyn Gal $^{2} \cdot$ Nathalie Ebran ${ }^{1} \cdot$ Josiane Otto $^{3} \cdot$ Delphine Borchiellini $^{3} \cdot$ Frederic Peyrade $^{3}$. \\ Emmanuel Chamorey ${ }^{2} \cdot$ Patrick Brest $^{4} \cdot$ Gérard Milano $^{1} \cdot$ Esma Saada-Bouzid $^{3}$
}

Published online: 4 June 2020

(C) Springer Science+Business Media, LLC, part of Springer Nature 2020

\section{Correction to: Invest New Drugs (2020) 38(1):160-171 https://doi.org/10.1007/s10637-019-00845-w}

Corrections are needed to the original version of this article. The in-text citations of tables and figures in the section "results" are not in sequential order. Moreover in section "results" (Associations between treatment outcome and SNPs), the reader should read "Almost half the patients $(43.5 \%, \mathrm{~N}=41)$ were in the low-risk group" and not "Almost half the patients $(43.5 \%, \mathrm{~N}=53)$ were in the lowrisk group".

The authors apologize for corrections and further state that changes to the text and the sequence of figures and tables does not affect the overall outcome of the study.

- Table 3 should show the univariate analysis for the 163 SNPs according to treatment outcome. Instead in the original paper it is presented in Table 4. For rs 13900 the alleles associated with recessive model are " $\mathrm{C} / \mathrm{C}$ or $\mathrm{C} / \mathrm{T}$ " and not " $\mathrm{C} / \mathrm{C}$ or $\mathrm{T} / \mathrm{T}$ " as written in the table.
- Table 4 should show the univariate analysis for the 163 SNPs according to toxicity, instead in the original paper it is presented in Table 3 .

- Table 5 should respectively show the weight of each SNP for multivariate predictive model according to treatment outcome and toxicity. Moreover in "Treatment outcome" the modality for rs13900 is $\mathrm{T} / \mathrm{T}$ and not $\mathrm{C} / \mathrm{T}$.

- Table 6 should show the classification of patients based on risk group and risk evaluation of each group. For a better reading of table, the results "Treatment Outcome" and "Toxicity" have been reversed.

- Figure 1a should show the performance of the treatment predictive model, instead in the original paper it is presented in the Fig. $1 \mathrm{~b}$

- Figure $1 \mathrm{~b}$ should show the performance of the toxicity predictive model, instead in the original paper it is presented in the Fig. 1a

The online version of the original article can be found at https://doi.org/ 10.1007/s10637-019-00845-w

Gérard Milano

gerard.milano@nice.unicancer.fr

Esma Saada-Bouzid

esma.saada-bouzid@nice.unicancer.fr

1 Centre Antoine Lacassagne, Oncopharmacology Unit, University Côte d'Azur, F-06189 Nice, France

2 Centre Antoine Lacassagne, Epidemiology and Biostatistics Department, University Côte d'Azur, F-06189 Nice, France

3 Centre Antoine Lacassagne, Medical Oncology Department, University Côte d'Azur, F-06189 Nice, France

4 Centre Antoine Lacassagne, CNRS, Inserm, Ircan, FHU-Oncoage, University Côte d'Azur, F-06189 Nice, France 
Table 3 Univariate analysis for the 163 SNPs according to treatment outcome

\begin{tabular}{|c|c|c|c|c|c|c|c|}
\hline $\begin{array}{l}\text { Variable } \\
\text { SNPs }\end{array}$ & Model & Genotype & $\mathrm{RC}$ or RP & $\mathrm{SD}$ or $\mathrm{PR}$ & OR & C195\% & $P$ value \\
\hline \multirow[t]{2}{*}{ rs1799983 (NOS3) } & \multirow[t]{2}{*}{ Dominant } & $\mathrm{G} / \mathrm{G}$ & $13(26.5)$ & $22(48.9)$ & 1 & Referent & \\
\hline & & $\mathrm{G} / \mathrm{T}$ or $\mathrm{T} / \mathrm{T}$ & $36(73.5)$ & $23(51.1)$ & 0.37 & {$[0.15-0.91]$} & 0.026 \\
\hline \multirow[t]{2}{*}{ rs4845618 (IL6R) } & \multirow[t]{2}{*}{ Dominant } & $\mathrm{T} / \mathrm{T}$ & 14 (28.6) & $23(51.1)$ & 1 & Referent & \\
\hline & & $\mathrm{G} / \mathrm{T}$ or $\mathrm{G} / \mathrm{G}$ & $35(71.4)$ & $22(48.9)$ & 0.38 & {$[0.16-0.91]$} & 0.027 \\
\hline \multirow[t]{2}{*}{ rs3212227 (IL12B) } & \multirow[t]{2}{*}{ Dominant } & $\mathrm{T} / \mathrm{T}$ & $23(46.9)$ & $31(68.9)$ & 1 & Referent & \\
\hline & & $\mathrm{G} / \mathrm{T}$ or $\mathrm{G} / \mathrm{G}$ & $26(53.1)$ & $14(31.1)$ & 0.39 & {$[0.16-0.94]$} & 0.033 \\
\hline \multirow[t]{2}{*}{ rs419598 (IL1RN) } & \multirow[t]{2}{*}{ Dominant } & $\mathrm{T} / \mathrm{T}$ & $33(67.3)$ & $18(40)$ & 1 & Referent & \\
\hline & & $\mathrm{C} / \mathrm{T}$ or $\mathrm{C} / \mathrm{C}$ & $16(32.7)$ & $27(60)$ & 3.09 & {$[1.30-7.32]$} & 0.008 \\
\hline \multirow[t]{2}{*}{ rs2280964 (CXCR3) } & \multirow[t]{2}{*}{ Dominant } & $\mathrm{C} / \mathrm{C}$ & $33(67.3)$ & $39(86.7)$ & 1 & Referent & \\
\hline & & $\mathrm{C} / \mathrm{T}$ or $\mathrm{T} / \mathrm{T}$ & $16(32.7)$ & $6(13.3)$ & 0.31 & {$[0.10-0.92]$} & 0.031 \\
\hline \multirow[t]{2}{*}{ rs 13900 (CCL2) } & \multirow[t]{2}{*}{ Recessive } & $\mathrm{C} / \mathrm{C}$ or $\mathrm{C} / \mathrm{T}$ & $49(100)$ & $41(91.1)$ & 1 & Referent & \\
\hline & & $\mathrm{T} / \mathrm{T}$ & $0(0)$ & $4(8.9)$ & - & {$[--]$} & $0.048^{*}$ \\
\hline \multirow[t]{2}{*}{ rs4586 (CCL2) } & \multirow[t]{2}{*}{ Recessive } & $\mathrm{T} / \mathrm{T}$ or $\mathrm{C} / \mathrm{T}$ & 47 (95.9) & $37(82.2)$ & 1 & Referent & \\
\hline & & $\mathrm{C} / \mathrm{C}$ & $2(4.1)$ & $8(17.8)$ & 5.08 & [0.98-26.25] & 0.047 \\
\hline \multirow[t]{2}{*}{ rs1024611 (CCL2) } & \multirow[t]{2}{*}{ Recessive } & $\mathrm{A} / \mathrm{A}$ or $\mathrm{A} / \mathrm{G}$ & $49(100)$ & $41(91.1)$ & 1 & Referent & \\
\hline & & $\mathrm{G} / \mathrm{G}$ & $0(0)$ & $4(8.9)$ & - & {$[--]$} & $0.048^{*}$ \\
\hline \multicolumn{8}{|l|}{ Patients and tumor characteristics } \\
\hline Age & & & $65.3(10.3)$ & $67.4(9.5)$ & 1.02 & {$[0.98-1.066]$} & 0.31 \\
\hline \multirow[t]{2}{*}{ Gender } & & Female & $18(42.9)$ & $22(48.9)$ & 1 & Referent & \\
\hline & & Male & $28(57.1)$ & $23(51.1)$ & 1.43 & {$[0.589-3.466]$} & 0.419 \\
\hline \multirow[t]{2}{*}{ Tabaco } & & No & $12(24.5)$ & $12(26.7)$ & 1 & Referent & \\
\hline & & Yes & $37(75.5)$ & $33(73.3)$ & 0.89 & {$[0.346-2.298]$} & 0.809 \\
\hline \multirow[t]{5}{*}{ Histology } & & HNSCC & $6(12.2)$ & $7(15.6)$ & 1 & Referent & \\
\hline & & NSCLC & $24(49)$ & $24(53.3)$ & 0.85 & {$[0.24-3.00]$} & 0.806 \\
\hline & & Melanoma & $9(18.4)$ & $3(6.7)$ & 0.28 & {$[0.05-1.62]$} & 0.149 \\
\hline & & Renal cell carcinoma & $6(12.2)$ & $8(17.8)$ & 1.14 & {$[0.24-5.38]$} & 0.863 \\
\hline & & Other & $4(8.2)$ & $3(6.7)$ & 0.64 & {$[0.09-4.25]$} & 0.640 \\
\hline \multirow[t]{2}{*}{ Radiotherapy } & & No & $18(36.7)$ & $12(26.7)$ & 1 & Referent & \\
\hline & & Yes & $31(53.1)$ & $33(73.3$ & 1.59 & {$[0.65-3.92]$} & 0.297 \\
\hline \multirow[t]{2}{*}{ Number of lines before recurrence } & & $0-1$ & $31(63.3)$ & $32(71.1)$ & 1 & Referent & \\
\hline & & $\geq 2$ & $18(36.7)$ & $13(28.9)$ & 0.7 & {$[0.28-1.69]$} & 0.419 \\
\hline
\end{tabular}

* OR not calculable replaced by Fisher's test; 
Table 4 Univariate analysis for the 163 SNPs according to toxicity.

\begin{tabular}{|c|c|c|c|c|c|c|c|}
\hline $\begin{array}{l}\text { Variable } \\
\text { SNPs }\end{array}$ & Model & Genotype & $\operatorname{IrAe}<3$ & $\operatorname{IrAe} \geq 3$ & OR & C195\% & $p$ value \\
\hline \multirow[t]{2}{*}{ rs246079 (UNG) } & \multirow[t]{2}{*}{ Dominant } & $\mathrm{A} / \mathrm{A}$ & $21(26.6)$ & $12(80)$ & 1 & Referent & \\
\hline & & $\mathrm{A} / \mathrm{G}$ or $\mathrm{G} / \mathrm{G}$ & $58(73.4)$ & $3(20)$ & 0.09 & {$[0.02-0.36]$} & $<0.001$ \\
\hline \multirow[t]{2}{*}{ rs10964859 (IFNW1) } & \multirow[t]{2}{*}{ Recessive } & $\mathrm{C} / \mathrm{C}$ or $\mathrm{C} / \mathrm{G}$ & $73(92.4)$ & $10(66.7)$ & 1 & Referent & \\
\hline & & $\mathrm{G} / \mathrm{G}$ & $6(7.6)$ & $5(33.3)$ & 6.08 & [1.52-24.33] & 0.014 \\
\hline \multirow[t]{2}{*}{ rs4143815 (PD-L1) } & \multirow[t]{2}{*}{ Recessive } & $\mathrm{G} / \mathrm{G}$ or $\mathrm{G} / \mathrm{C}$ & $73(92.4)$ & $9(60)$ & 1 & Referent & \\
\hline & & $\mathrm{C} / \mathrm{C}$ & $6(7.6)$ & $6(40)$ & 8.11 & [2.09-31.40] & 0.003 \\
\hline \multirow[t]{2}{*}{ rs12979860 (IFNL4) } & \multirow[t]{2}{*}{ Recessive } & $\mathrm{C} / \mathrm{C}$ or $\mathrm{C} / \mathrm{T}$ & $67(84.8)$ & $9(60)$ & 1 & Referent & \\
\hline & & $\mathrm{T} / \mathrm{T}$ & $12(15.2)$ & $6(40)$ & 3.72 & [1.09-12.68] & 0.036 \\
\hline \multirow[t]{2}{*}{ rs3087243 (CTLA4) } & \multirow[t]{2}{*}{ Recessive } & $\mathrm{A} / \mathrm{A}$ or $\mathrm{A} / \mathrm{G}$ & $62(78.5)$ & $8(53.3)$ & 1 & Referent & \\
\hline & & $\mathrm{G} / \mathrm{G}$ & $17(21.5)$ & $7(46.7)$ & 3.19 & [1.01-10.27] & 0.048 \\
\hline \multirow[t]{2}{*}{ rs11571302 (CTLA4) } & \multirow[t]{2}{*}{ Recessive } & $\mathrm{T} / \mathrm{T}$ or $\mathrm{G} / \mathrm{T}$ & $65(82.3)$ & $8(53.3)$ & 1 & Referent & \\
\hline & & $\mathrm{G} / \mathrm{G}$ & $14(17.7)$ & $7(46.7)$ & 4.06 & {$[1.23-13.38]$} & 0.018 \\
\hline \multirow[t]{2}{*}{ rs7565213 (CTLA4) } & \multirow[t]{2}{*}{ Recessive } & $\mathrm{A} / \mathrm{A}$ or $\mathrm{A} / \mathrm{G}$ & $64(81)$ & $8(53.3)$ & 1 & Referent & \\
\hline & & $\mathrm{G} / \mathrm{G}$ & $15(19)$ & $7(46.7)$ & 3.73 & [1.14-12.19] & 0.026 \\
\hline \multicolumn{8}{|l|}{ Patients and tumor characteristics } \\
\hline Age & & & $65.5(10)$ & $70.5(8.4)$ & 1.062 & {$[0.99-1.13]$} & 0.075 \\
\hline \multirow[t]{2}{*}{ Gender } & & Female & $25(31.6)$ & $6(40)$ & 1 & Referent & \\
\hline & & Male & $54(68.4)$ & $9(60)$ & 0.69 & {$[0.21-2.21]$} & 0.53 \\
\hline \multirow[t]{2}{*}{ Tobacco } & & No & $20(25.3)$ & $4(26.7)$ & 1 & Referent & \\
\hline & & Yes & $59(74.7)$ & $11(73.3)$ & 0.93 & {$[0.26-3.34]$} & 0.91 \\
\hline \multirow[t]{5}{*}{ Histology } & & $\mathrm{HNSCC}^{*}$ & $11(13.9)$ & $2(13.3)$ & 1 & Referent & \\
\hline & & NSCLC $^{* *}$ & $41(51.9)$ & $7(46.7)$ & 0.94 & {$[0.16-5.36]$} & 0.94 \\
\hline & & Melanoma & $11(13.9)$ & $1(6.7)$ & 0.50 & {$[0.03-6.69]$} & 0.59 \\
\hline & & Renal cell carcinoma & $11(13.9)$ & $3(20)$ & 1.50 & {$[0.20-11.25]$} & 0.68 \\
\hline & & Other & $5(6.3)$ & $2(13.3)$ & 2.20 & {$[0.22-21.33]$} & 0.48 \\
\hline \multirow[t]{2}{*}{ Radiotherapy } & & No & $23(29.1)$ & $7(46.7)$ & 1 & Referent & \\
\hline & & Yes & $56(70.9)$ & $8(53.3)$ & 0.47 & {$[0.14-1.48]$} & 0.18 \\
\hline \multirow[t]{2}{*}{ Number of lines before recurrence } & & $0-1$ & $53(67.1)$ & $10(66.7)$ & 1 & Referent & \\
\hline & & $\geq 2$ & $26(32.9)$ & $5(33.3)$ & 1.01 & {$[0.30-3.37]$} & 0.97 \\
\hline
\end{tabular}

\footnotetext{
${ }^{*}$ HNSCC: Head and neck squamous cell carcinoma; ${ }^{* *}$ NSCLC: non-small cell lung carcinoma
} 
Table 5 Weight of each variable for multivariate predictive model

\begin{tabular}{llllll}
\hline rs ID (Gene) & Chr & Ancestral allele & Minor Allele Frequency & Modality & Weight \\
\hline Treatment outcome & & & & & \\
rs13900 (CCL2) & 17 & $\mathrm{C}$ & 0.5 & $\mathrm{~T} / \mathrm{T}$ & 347 \\
rs1799983 (NOS3) & 7 & $\mathrm{G}$ & 0.4 & $\mathrm{G} / \mathrm{G}$ & 133 \\
rs3212227 (IL12B) & 5 & $\mathrm{~T}$ & 0.5 & $\mathrm{~T} / \mathrm{T}$ & 133 \\
rs419598 (IL1RN) & 2 & $\mathrm{~T}$ & 0.42 & $\mathrm{C} / \mathrm{T}$ or C/C & 133 \\
rs2280964 (CXCR3) & $\mathrm{X}$ & $\mathrm{C}$ & 0.49 & $\mathrm{C} / \mathrm{C}$ & 119 \\
rs4845618 (IL6R) & 1 & $\mathrm{G}$ & 0.49 & $\mathrm{~T} / \mathrm{T}$ & 102 \\
rs4586 (CCL2) & 17 & $\mathrm{C}$ & $\mathrm{C} / \mathrm{C}$ & 32 \\
Toxicity & & & & $\mathrm{G} / \mathrm{G}$ & 243 \\
rs10964859 (IFNW1) & 9 & $\mathrm{C}$ & $\mathrm{G}$ & $\mathrm{A} / \mathrm{A}$ & 243 \\
rs246079 (UNG) & 12 & $\mathrm{G}$ & 0.37 & $\mathrm{G} / \mathrm{G}$ & 187 \\
rs3087243 (CTLA4) & 2 & $\mathrm{G}$ & 0.41 & $\mathrm{C} / \mathrm{C}$ & 175 \\
rs4143815 (PDL-1) & 9 & $\mathrm{G}$ & 0.45 & $\mathrm{~T} / \mathrm{T}$ & 152 \\
rs12979860 (IFNL4) & 19 & $\mathrm{~T}$ & 0.33 & & \\
\hline
\end{tabular}

Table 6 Classification of patients based on risk group and risk

Treatment Outcome evaluation of each group

\begin{tabular}{|c|c|c|c|c|c|}
\hline Risk group & Total n $(\%)$ & $\mathrm{RC}$ or PR & $\mathrm{SD}$ or $\mathrm{PD}$ & Odds Ratio (CI 95\%) & $\mathrm{p}$ \\
\hline Low risk & $41(43.5 \%)$ & $33(80.5 \%)$ & $8(19.5 \%)$ & 1.0 referent & 0.006 \\
\hline Intermediate risk & $27(29 \%)$ & $13(48 \%)$ & $14(52 \%)$ & $4.42[1.48-13.37]$ & \\
\hline High risk & $26(27.5 \%)$ & $3(11.5 \%)$ & $23(88.5 \%)$ & $31.62[7.36-135.91]$ & $<0.001$ \\
\hline \multicolumn{6}{|l|}{ Toxicity } \\
\hline & & No IrAE & IrAE & & \\
\hline Low risk & $70(74.5 \%)$ & $67(95.7 \%)$ & $3(4.3 \%)$ & 1.0 referent & $<0.001$ \\
\hline High risk & $24(25.5 \%)$ & $12(50 \%)$ & $12(50 \%)$ & 22.33 [5.31-93.87] & \\
\hline
\end{tabular}


a Treatment outcome

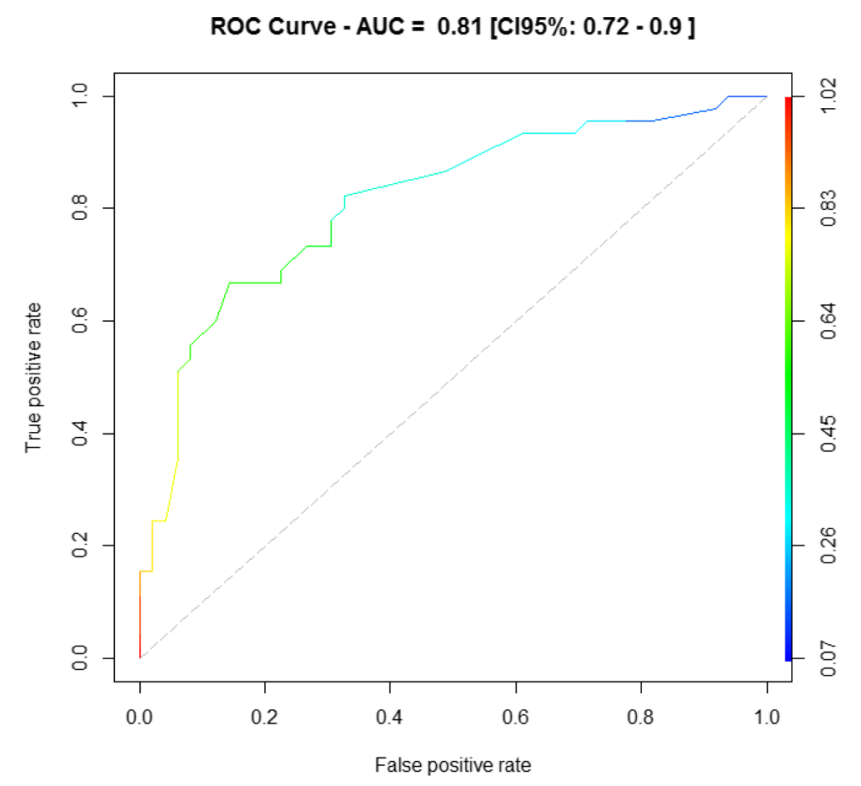

b Toxicity

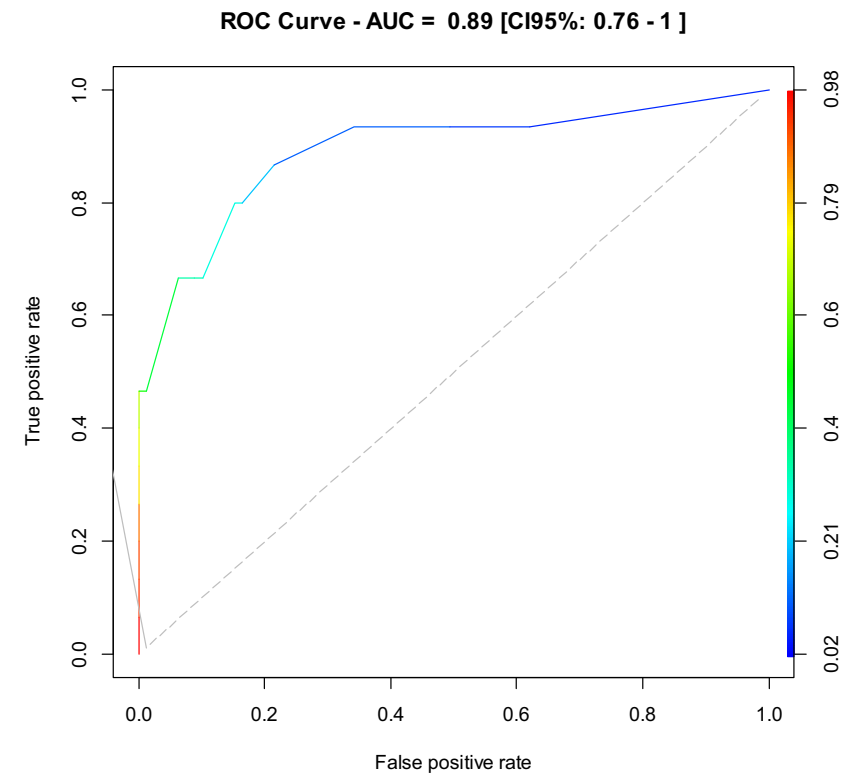

Fig. 1 ROC curves representation and AUC estimation for predictive model. a Treatment outcome, $\mathbf{b}$ Toxicity 
a Treatment outcome

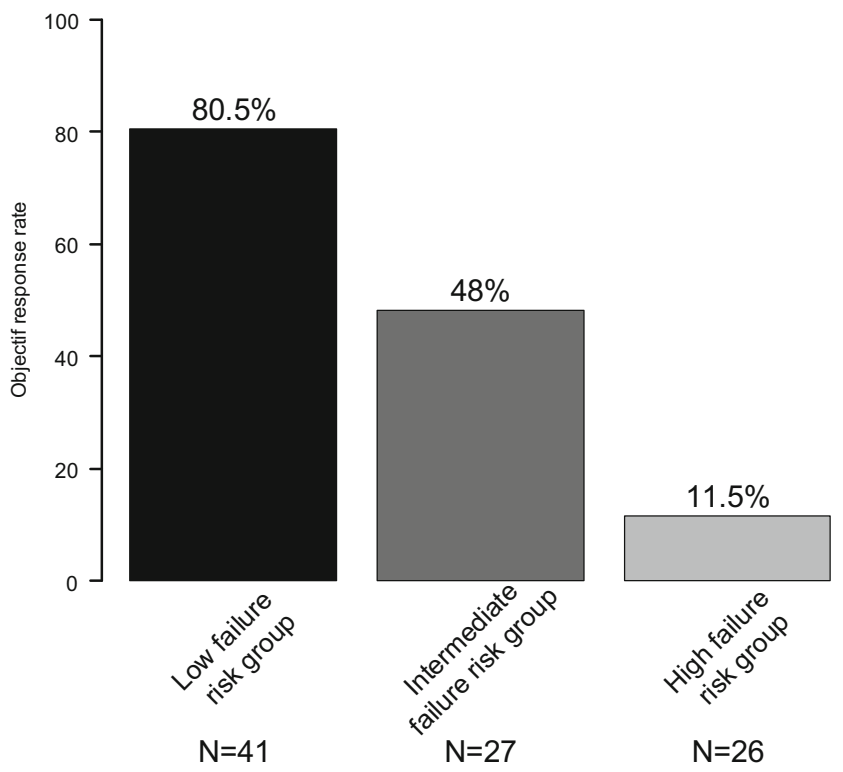

b Toxicity

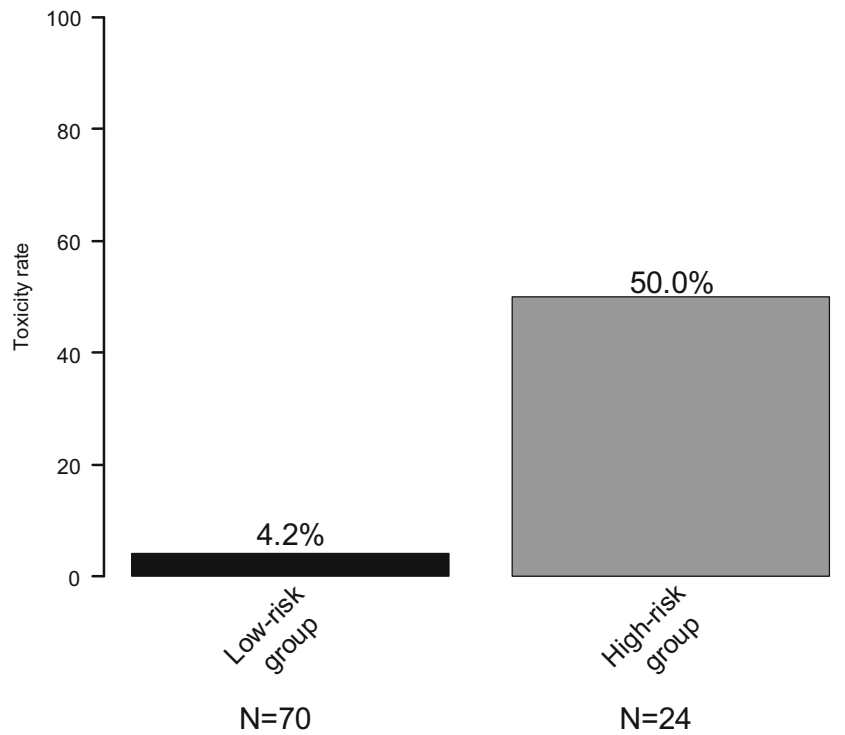

Fig. 2 Patients distribution in the different risk groups according on the predictive model. a Treatment outcome, b Toxicity

Publisher's note Springer Nature remains neutral with regard to jurisdictional claims in published maps and institutional affiliations. 